\title{
Research on Wavelet Transform Blind Equalizer Based on Different Place
}

\author{
HAN Yingge ${ }^{1, a^{*}}$, LI Baokun ${ }^{2, b}$ and Zhang Lin ${ }^{1, c}$ \\ ${ }^{1}$ College of Electrical Engineering and Information, Anhui University of Science and Technology, \\ Huainan 232001, China \\ ${ }^{2}$ College of Mechanical Engineering, Anhui University of Science and Technology, Huainan \\ 232001, China \\ a han_ying_ge@126.com, ${ }^{b}$ li_bao_kun@126.com, ${ }^{c}$ zl8081676@qq.com
}

\begin{abstract}
Keywords: Constant modulus algorithm, decision feedback equalizer, balance orthogonal multi wavelet transform.

Abstract. In order to improve the convergence speed of adaptive equalizer, orthogonal wavelet transform has been introduced into adaptive equalizer. The orthogonal wavelet transform are placed in the front of the equalizer (forward filter) in the existing literature, which will reduce the performance of equalizer. In order to study the equalizer performance under the different placement of wavelet transform, based on the Constant Modulus Algorithm Decision Feedback Equalizer (CMA-DFE) as an example, the forward balance orthogonal multi-wavelet transform based CMA-DFE (MWT-CMA-DFE), the feedback balance orthogonal multi-wavelet transform based CMA-DFE(FMWT-CMA-DFE) and the dual balance orthogonal multi-wavelet transform based CMA-DFE (DMWT-CMA-DFE) are proposed and analyzed respectively. Moreover, the complexities of the proposed equalizers are analyzed. Simulation tests with underwater acoustic channel have indicated that the DMWT-CAM-DFE not only has the faster the performances of convergence and tracking the time vary channel but also can eliminate phase rotation compared with CMA-DFE, MWT-CMA-DFE and FMWT-CMA-DFE.
\end{abstract}

\section{Introduction}

In the underwater communication systems, constant modulus algorithm based decision feedback blind equalizer (CMA-DFE) is an effective and popular method to eliminate inter-symbol interference (ISI) at the receiver end. However, it suffers from the slow convergence speed [1-2]. In order to improve the convergence speed, the transform domain method has been proposed, such as walsh-hadamard transform, karhumen-loeve transform, discrete fourier transform[11] and discrete cosine transform, etc. In recent years, with the development of wavelet transform theory, a new transform domain equalizer has been studied. The convergence speed of the equalizer can be improved, while the normalized orthogonal wavelet transform is used for the input signal of equalizer [3-10]. In [3], the momentum term and orthogonal wavelet transform based blind equalization algorithm is proposed, in which the momentum term and orthogonal wavelet transform are introduced into the blind equalization (BE) algorithm, and the convergence speed of the equalizer is improved by means of normalizing for the equalizer input signal and the momentum. An orthogonal wavelet transform based LE+DD algorithm is proposed in [4]. Compared with the single wavelet, the multi-wavelet consists of several scaling functions and its support is no speed, overlapping at any scale. So it can overcome the boundary effect effectively. In [5], a balanced orthogonal multi wavelet transform based BE algorithm is proposed. In order to further improve the convergence, the wavelet transform blind equalization algorithm based on ant colony optimization is studied in[6].Although the existing algorithms can improve the convergence speed of equalizer, the orthogonal wavelet transform are placed before the transverse filter (forward filter), which will affect the performance. In this paper, the effect of wavelet transform location for the performance of the equalizer is analyzed. 


\section{CMA-DFE}

The structure of CMA-DFE[2] is shown in Fig.1. It consists of two filter components that the forward filter and feedback ones. The forward filter is the same as the transversal filter of the linear equalization, in which the output of the channel is considered as the input of the forward filter. The feedback filter component is used to counteract the interference from the previous symbol, in which the decision device output signal is considered as the input of feedback filter. $\mathrm{Qu}, \mathrm{g}(\cdot), \mathrm{C}$ and $n(n)$ denote the decision device, the nonlinear transformation device, the channel frequency response and the additive noise of the channel respectively.

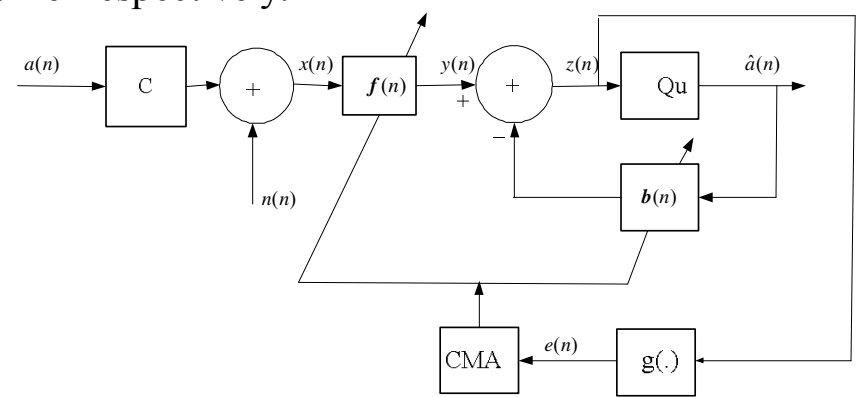

Fig. 1 The structure of CMA-DFE

As shown in Fig.1. the tapping coefficient vector of the forward filter and feedback filter is the the $\boldsymbol{f}(n)$ and $\boldsymbol{b}(n)$ respectively, which can be represented as:

$$
\begin{aligned}
& \boldsymbol{f}(n)=\left[f(0) \quad, \quad f(1) \quad, \quad \mathrm{L} \quad, \quad f\left(N_{f}-1\right)\right]^{T}
\end{aligned}
$$

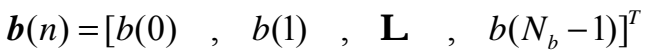

Where $N_{f}$ and $N_{b}$ means the weight length of the forward and feedback filter respectively.

Assume that the input recursion vector of the forward and feedback filter is $\boldsymbol{X}(n)$ and $\boldsymbol{A}(n)$. Thus, $\boldsymbol{X}(n)$ and $\boldsymbol{A}(n)$ can be expressed as:

$$
\begin{aligned}
& \boldsymbol{X}(n)=\left[\begin{array}{llll}
x(n) & , & x(n-1), & \mathrm{L} \quad, \quad x\left(n-N_{f}+1\right)
\end{array}\right]^{T} \\
& \boldsymbol{A}(n)=\left[\begin{array}{lll}
\hat{a}(n), & \hat{a}(n-1), \quad \mathrm{L} \quad, \quad \hat{a}\left(n-N_{f}+1\right)
\end{array}\right]^{T}
\end{aligned}
$$

According to structure of CMA-DFE in the Fig.1, the decision device input is given as

$$
z(n)=\sum_{i=0}^{N_{f}-1} f(n) x(n-i)-\sum_{i=0}^{N_{b}-1} b(n) \hat{a}(n-i)=\boldsymbol{f}^{T}(n) \boldsymbol{X}(n)-\boldsymbol{b}^{T}(n) \boldsymbol{A}(n)
$$

where $\hat{a}(n)$ denotes the decision device output.

The CMA is used to adjust the weights of both the forward and feedback filters in the minimum mean square criteria, the weight coefficients update formulas can be shown as

$$
\left\{\begin{array}{l}
\boldsymbol{f}(n+1)=\boldsymbol{f}(n)-\mu_{1} e(n) \boldsymbol{y}^{*}(n) z(n) \\
\boldsymbol{b}(n+1)=\boldsymbol{b}(n)+\mu_{2} e(n) \hat{\boldsymbol{a}}^{*}(n) z(n)
\end{array}\right.
$$

where $\mu_{1}$ and $\mu_{2}$ denotes the step size, $e(n)$ stand for the error function, and

$$
e(n)=|z(n)|^{2}-R_{2}
$$

$R_{2}=\mathrm{E}\left[|a(n)|^{4} /|a(n)|^{2}\right]$.The algorithm consisted of Eq.(1-7) is define as constant module based decision feedback BE algorithm(CMA-DFE). In the Eq.(6), $\mu_{1}$ and $\mu_{2}$ are constant, if the step size is small, the convergence is slow, if the step increases, the convergence becomes large. 


\section{DMWT-CMA-DFE}

\section{MWT-CMA-DFE}

Based on the studies in [3-9], the convergence speed of equalizer can be improved by normalizing orthogonal wavelet transform for the equalizer input signal, when the orthogonal wavelet transform is placed in the front of the forward filters. However, the performance of equalizer is affected for the placement of wavelet transform. In this section, the balanced orthogonal multi wavelet transform based constant modulus algorithm decision feedback blind equalizer (MWT-CMA-DFE)[4] is analyzed. Fig.2 gives the structure of MWT-CMA-DFE.

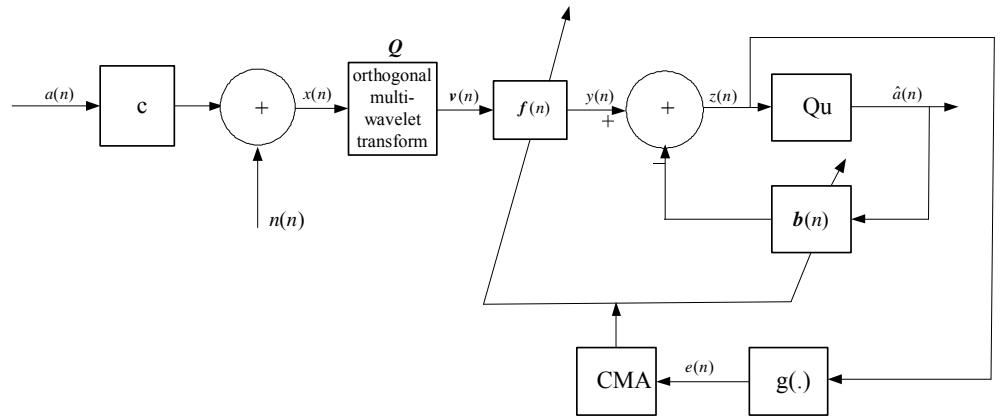

Fig.2 The structure of MWT-CMA-DFE

In Fig.2, the forward filter input signal vector is $\boldsymbol{X}(n)$, it can be expressed by Eq.(3). Assume that the output signal vector by orthogonal multi-wavelet transform is $\boldsymbol{v}(n)$, which can be formulated as

$$
\boldsymbol{v}(n)=\left[r_{1,0}(n), r_{1,1}(n), \mathrm{L}, r_{J, k_{J}}(n), s_{J, 0}(n), \mathrm{L} s_{J, k_{J}}(n)\right]^{T}
$$

where $\mathrm{J}$ represents the maximum scaling of wavelet decomposition, $k_{j}$ is the maximum translation of wavelet function under the scaling $j$, and $k_{j}$ can be given by $k_{j}=N / 2^{j}-1(\mathrm{j}=1,2, \ldots, \mathrm{J})$.

$$
\boldsymbol{v}(n)=\boldsymbol{Q X}(n)
$$

$\boldsymbol{Q}$ denote as the orthogonal multi wavelet transform matrix, $\boldsymbol{Q}$ can be given by

$$
\boldsymbol{Q}=\left[\boldsymbol{Q}_{1} ; \boldsymbol{Q}_{2} \boldsymbol{P}_{1} ; \boldsymbol{Q}_{2} \boldsymbol{P}_{2} \boldsymbol{P}_{1} ; \mathrm{L} ; \boldsymbol{Q}_{J} \boldsymbol{P}_{J-1} \mathrm{~L} \boldsymbol{P}_{2} \boldsymbol{P}_{1} ; \boldsymbol{P}_{J} \boldsymbol{P}_{J-1} \mathrm{~L} \boldsymbol{P}_{2} \boldsymbol{P}_{1}\right]^{[7]}
$$

it is a $\mathrm{N}_{\mathrm{f}} \times \mathrm{N}_{\mathrm{f}}$ orthogonal matrix. Therefore, under the minimum mean square criteria, the weight coefficients update formula of MWT-CMA-DFE can be shown as:

$$
\left\{\begin{array}{l}
\boldsymbol{f}(n+1)=\boldsymbol{f}(n)-\mu_{1} \boldsymbol{R}^{-1}(n) \boldsymbol{v}^{*}(n) e(n) z(n) \\
\boldsymbol{b}(n+1)=\boldsymbol{b}(n)+\mu_{2} e(n) \hat{\boldsymbol{a}}^{*}(n) z(n)
\end{array}\right.
$$

where $\boldsymbol{R}(n)=\operatorname{diag}\left[\sigma_{j, 0}^{2}(n), \sigma_{j, 1}^{2}(n), \mathrm{L}, \sigma_{J, k_{J}}^{2}(n), \sigma_{J+1,0}^{2}(n) \mathrm{L}, \sigma_{J+1, k_{J}}^{2}(n)\right], \sigma_{j, k}^{2}(n)$ and $\sigma_{J+1, k}^{2}(n)$ are the average power value of the $r_{j, k}(n)$ and $s_{J, k}(n)$ respectively, they can be obtained via the following formula recursively

$$
\left\{\begin{array}{c}
\sigma_{j, k}^{2}(n+1)=\beta \sigma_{j, k}^{2}(n)+(1-\beta)\left|r_{j, k}(n)\right|^{2} \\
\sigma_{J+1, k}^{2}(n+1)=\beta \sigma_{J+1, k}^{2}(n)+(1-\beta)\left|s_{J, k}(n)\right|^{2}
\end{array}\right.
$$

In summary, the Eq.(3) and (8-12) defined as a common balanced orthogonal multi wavelet transform based constant modulus decision feedback BE algorithm. In the algorithm, the orthogonal multi wavelet transform is introduced into CMA-DFE, and the convergence speed of the CMA-DFE can be improved by normalizing orthogonal wavelet transform for the input signal of the forward filter. 


\section{FMWT-CMA-DFE}

In Fig.2, when the orthogonal multi-wavelet transform is placed before the feedback filter, a feedback balanced orthogonal multi wavelet transform based constant modulus decision feedback blind equalizer (FMWT-CMA-DFE) is proposed. The equalizer structure is shown in Fig.3.

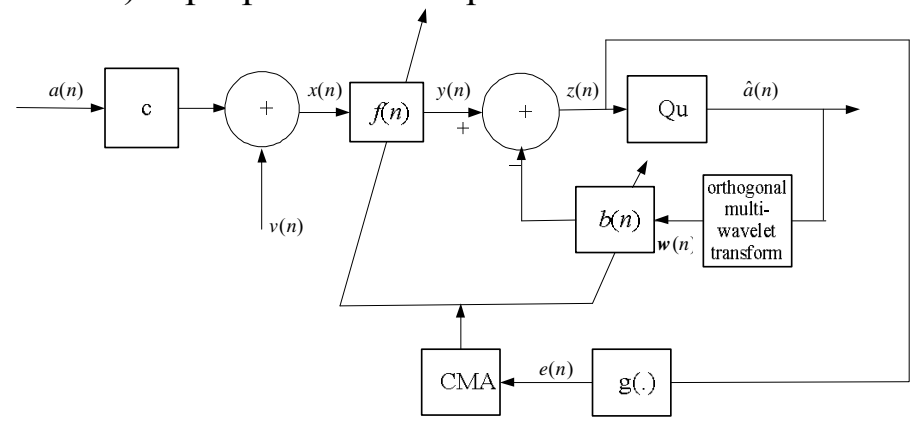

Fig.3 The structure of FMWT-CMA-DFE

In Fig.3

$$
\boldsymbol{w}(n)=\boldsymbol{G A}(n)
$$

where $G$ is the orthogonal multi-wavelet transformation matrix, it is a $\mathrm{N}_{\mathrm{b}} \times \mathrm{N}_{\mathrm{b}}$ orthogonal matrix, which is given by $\boldsymbol{G}=\left[\boldsymbol{G}_{1} ; \boldsymbol{G}_{2} \boldsymbol{P}_{1} ; \boldsymbol{G}_{2} \boldsymbol{P}_{2} \boldsymbol{P}_{1} ; \ldots ; \boldsymbol{G}_{J J} \boldsymbol{P}_{J J-1} \ldots \boldsymbol{P}_{2} \boldsymbol{P}_{1} ; \boldsymbol{G}_{2} \boldsymbol{P}_{1} ; \boldsymbol{G}_{2} \boldsymbol{P}_{2} \boldsymbol{P}_{1} ; \ldots ; \boldsymbol{G}_{J J} \boldsymbol{P}_{J J-1} \ldots \boldsymbol{P}_{2} \boldsymbol{P}_{1} ;\right] \quad J J$ represents the maximum scaling of wavelet decomposition. $\boldsymbol{A}(n)$ is the output recursive vector of the decision device, which is given by Eq.(4), $w(n)$ is the output recursive vector by orthogonal multi-wavelet transformation.

Similar as Eq.(11), under the minimum criteria, the weight coefficients update formula can be shown as

$$
\left\{\begin{array}{l}
\boldsymbol{f}(n+1)=\boldsymbol{f}(n)-\mu_{1} y^{*}(n) e(n) z(n) \\
\boldsymbol{b}(n+1)=\boldsymbol{b}(n)+\mu_{2} \boldsymbol{R}^{-1}(n) e(n) \boldsymbol{w}^{*}(n) z(n)
\end{array}\right.
$$

where $\boldsymbol{R} \boldsymbol{R}(n)=\operatorname{diag}\left[\sigma_{j, 0}^{2}(n), \sigma_{j, 1}^{2}(n), \sigma_{j, 2}^{2}(n), \mathbf{L}, \sigma_{J J, k_{J}}^{2}(n), \sigma_{J J+1,0}^{2}(n), \mathbf{L}, \sigma_{J J+1, k_{J}}^{2}(n)\right], \sigma_{j, k}^{2}(n)$ and $\sigma_{J J+1, k_{j}}^{2}(n)$ express the average power value of the $r_{j, k}(n)$ and $s_{J J, k}(n)$ respectively, which can be obtained by the following formula

$$
\left\{\begin{array}{c}
\hat{\sigma}_{j, k}^{2}(n+1)=\beta \hat{\sigma}_{j, k}^{2}(n)+(1-\beta)\left|r_{j, k}(n)\right|^{2} \\
\hat{\sigma}_{J J+1, k}^{2}(n+1)=\beta \hat{\sigma}_{J J+1, k}^{2}(n)+(1-\beta)\left|s_{J J, k}(n)\right|^{2}
\end{array}\right.
$$

we define the algorithm consisted of Eq.(4) and (13-15) as a feedback balanced orthogonal multi-wavelet transform based constant modulus decision feedback BE algorithm.

\section{DMWT-CMA-DFE}

In Fig. 1, when the orthogonal multi-wavelet transform is placed not only on the front of the forward filter but also on the front of the feedback filter, we define this equalizer as the dual balanced orthogonal multi wavelet transform based constant modulus decision feedback blind equalizer (DMWT-CMA-DFE), it's structure is shown as Fig.4. 


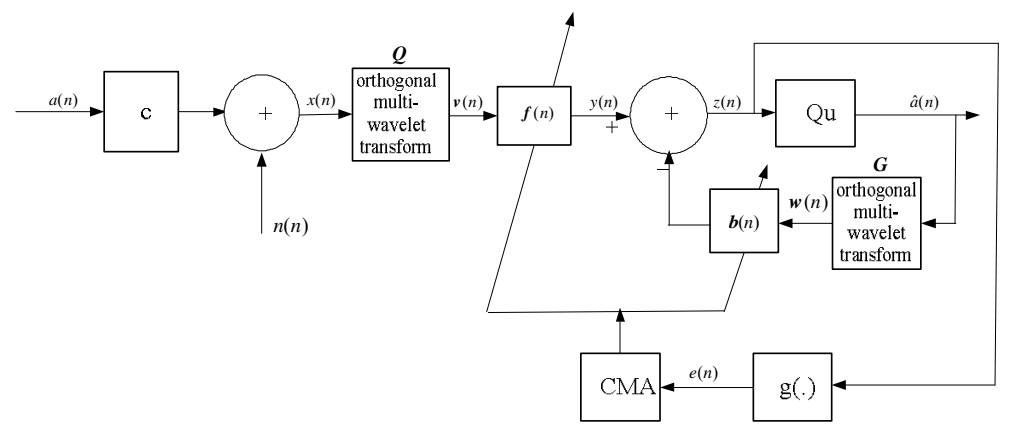

Fig.4 the structure of DMWT-CMA-DFE

In Fig.4

$$
\begin{aligned}
& \boldsymbol{v}(n)=\boldsymbol{Q} \boldsymbol{X}(n) \\
& \boldsymbol{w}(n)=\boldsymbol{G} \boldsymbol{A}(n)
\end{aligned}
$$

Also, under the minimum criteria, the weight coefficients update formula can be shown as:

$$
\left\{\begin{array}{l}
\boldsymbol{f}(n+1)=\boldsymbol{f}(n)-\mu_{1} \boldsymbol{R}^{-1}(n) \boldsymbol{v}^{*}(n) e(n) z(n) \\
\boldsymbol{b}(n+1)=\boldsymbol{b}(n)+\mu_{2} \boldsymbol{R}^{-1}(n) \boldsymbol{w}^{*}(n) e(n) z(n)
\end{array}\right.
$$

The algorithm consist of Eq.(16 18) is defined as a dual balanced orthogonal multi-wavelet transform based constant modulus decision feedback BE algorithm.

\section{The comparison of Wavelet Transform Blind Equalizer Based on Different Place}

In Fig. 2, before adjusting forward filter weight coefficients, MWT-CMA - DFE firstly carry out the normalized orthogonal multi-wavelet transform for forward filter's input, but the forward filters input signals are the sum of signal and noise, the essence is that the normalized orthogonal multi wavelet transform for signal and noise respectively. In Fig.3, before adjusting feedback filter weight coefficients, FMWT - CMA - DFE firstly carry on the normalized orthogonal multi wavelet transform for the feedback filter's input. However, the feedback filter input signal is equal to the decision output, which is basically closing to the original launch signal, so the essence of FMWT - CMA - DFE is does orthogonal multi wavelet transform for the input signal only. In Fig. 4, before adjusting the weight coefficients of forward filters and feedback filters, DMWT - CMA - DFE firstly do the normalized orthogonal multi wavelet transform for the their input respectively. Therefore, for the computational complexity, FMWT - CMA - DFE is the lowest, DMWT - CMA - DFE is the highest, and the MWT CMA - DFE is medium.

\section{Simulation and analysis}

To verify the effect of orthogonal wavelet transform location on the performance of equalizer, the computer simulation is carried out for the CMA-DFE, MWT-CMA-DFE, FMWT-CMA-DFE and DMWT-CMA-DFE. In this simulation, the source signal is 16-QAM, the signal to noise ratio is $25 \mathrm{~dB}$, the length of forward filter is 33 , the length of feedback filter is $16, \beta$ is equal to 0.9 , the initial value of $\boldsymbol{R}(n)$ and $\boldsymbol{R} \boldsymbol{R}(n)$ is equal to 1.

\section{Simulation with mixed phase underwater acoustic channel}

The channel impulse response is given by

$$
\mathrm{C}=\left[\begin{array}{lll}
1, & 0,0.3 \mathrm{e}-0.7 \mathrm{i}, \quad 0, \quad 0,0.2 \mathrm{e}-0.8 \mathrm{i}
\end{array}\right]
$$

the value of the others simulation parameters are shown in Tab.1. The simulation results are shown in Fig.5. 
Tab.1 The value of simulation parameters

\begin{tabular}{|c|l|c|c|c|}
\hline \multirow{2}{*}{ Equalizer } & \multicolumn{2}{|c|}{ Size-step } & \multicolumn{2}{|c|}{ Initial weight } \\
\cline { 2 - 3 } & forward filter & feedback filter & $\begin{array}{c}\text { Forward } \\
\text { filter }\end{array}$ & Feedback filter \\
\hline CMA-DFE & 0.00002 & 0.00002 & $\begin{array}{c}\text { the first tap } \\
\text { is } 1 \text {, the } \\
\text { others is } 0\end{array}$ & $\begin{array}{l}\text { the sixteen tap } \\
\text { is } 1 \text {, the others } \\
\text { is } 0\end{array}$ \\
\hline MWT-CMA-DFE & 0.001 & 0.001 & 0.002 & \\
\hline FMWT-CMA-DFE & 0.0005 & 0.003 & \\
\hline DMWT-CMA-DFE & 0.00004 & \multicolumn{2}{|c|}{} &
\end{tabular}

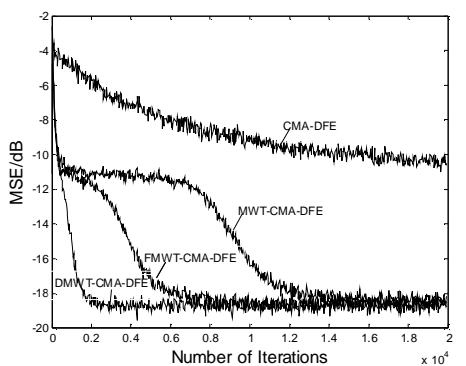

(a) Learning curse of MSE

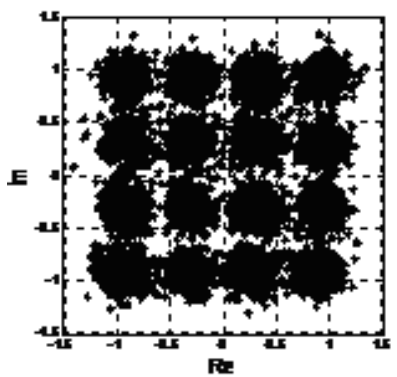

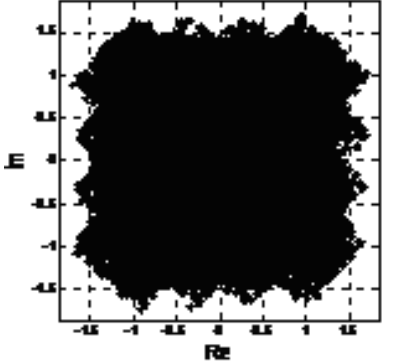

(b) Input of equalizer

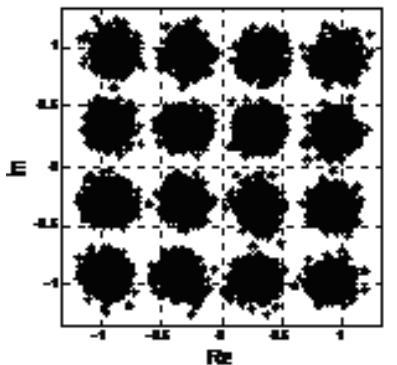

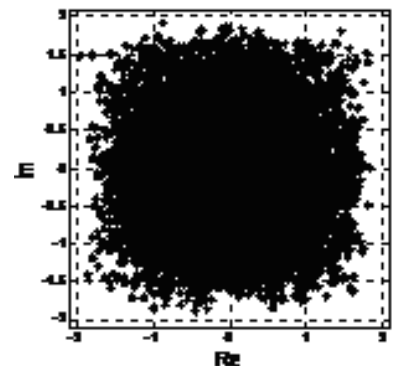

(c) Output of CMA-DFE

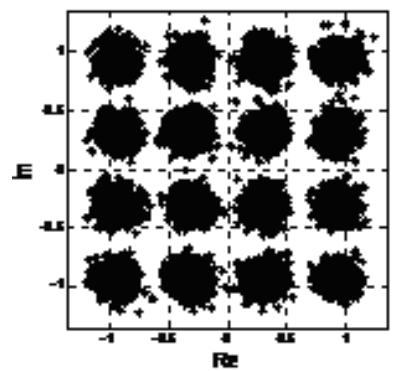

(d) Output of MWT-CMA-DFE (e) Output of FWT-CMA-DFE (f) Output of DMWT-CMA-DFE

Fig. 5 Simulation of mixed phase channel

Fig. 5 shows that the convergence speed of DMWT-CMA-DFE is the fastest, it is faster nearly 17,000 steps than CMA-DFE, nearly 12,000 steps than MWT-CMA-DFE, nearly 7,000 steps than FMWT-CMA-DFE.The residual mean square error of DMWT-CMA-DFE is smaller about 8dB than CMA-DFE and that is nearly same with MWT-CMA-DFE and FMWT-CMA-DFE. Fig.5 $(b \sim \mathrm{f})$ give the constellation diagram of equalization before and after, the figure shows that the eye diagram of DMWT - CMA - DFE after equalization is more compact and eliminates the phase rotation.

\section{Simulation with time-varying channel}

In order to construct a time-varying channel, the $\mathrm{c} 1$ and $\mathrm{c} 2$ channel is combined to simulate a time-varying channel. In the iterative initial stage, the signal is transmitted in the $\mathrm{cl}$ channel, the $\mathrm{cl}$ channel can be expressed as

$$
\mathrm{cl}=\left[\begin{array}{llll}
0.3132 & -0.1040 & 0.8908 & 0.3134
\end{array}\right]
$$

when iterative stage is equal to 10000 , the channel change for $\mathrm{c} 2$, it can be shown as

$$
\mathrm{c} 2=\left[\begin{array}{lllll}
1 & 0 & 0.498+i 0.0435 & 0 & 0.2955+i 0.0522
\end{array}\right]
$$

the others simulation parameters are shown in Tab.2.The result of simulations is shown in Fig.6.

\begin{tabular}{|c|c|c|c|c|}
\hline \multirow[b]{2}{*}{ Equalizer } & \multicolumn{2}{|c|}{ size-step } & \multicolumn{2}{|c|}{ Initial weight } \\
\hline & $\begin{array}{c}\text { forward } \\
\text { filter }\end{array}$ & feedback filter & Forward filter & Feedback filter \\
\hline CMA-DFE & 0.00005 & 0.00005 & \multirow{4}{*}{$\begin{array}{c}\text { the first tap is } \\
1 \text {, the others is } \\
0 .\end{array}$} & \multirow{4}{*}{$\begin{array}{l}\text { the sixteen tap } \\
\text { is } 1 \text {, the others } \\
\text { is } 0 .\end{array}$} \\
\hline MWT-CMA-DFE & 0.0001 & 0.0001 & & \\
\hline FMWT-CMA-DFE & 0.0001 & 0.0004 & & \\
\hline DMWT-CMA-DFE & 0.0007 & 0.0008 & & \\
\hline
\end{tabular}

Tab.2 The value of simulation parameters 


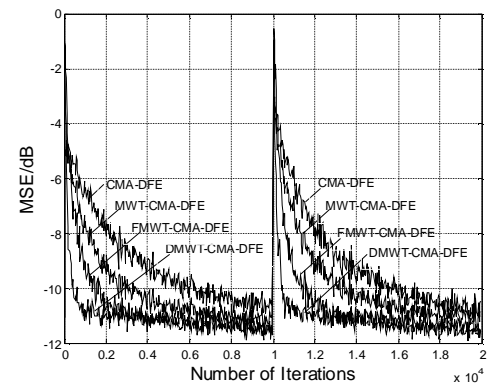

Fig.6 Learning curse of MSE

The Fig. 6 shows, when iterative stage is equal to 10000, the channel change, the mean square error suddenly becomes larger, the equalizer can converge again. This suggests that these algorithms have the some ability to track a timely channel. The other, compared with the FMWT - CMA - DFE, MWT - CMA - DFE and CMA - DFE, DMWT - CMA - DFE has faster the ability to track the time-varying channel.

\section{Summary}

Convergence speed and mean square error is the major index to measure the communication system performance. The existing design of adaptive equalizer based on orthogonal wavelet transform places orthogonal wavelet transform before the forward (transverse) filter, which affects the performance. In order to study the effect of orthogonal multi-wavelet transform location on the equalizer performance, the paper selects the constant module algorithm based decision feedback blind equalizer as an example. According to the different locations of orthogonal multi-wavelet transform, three equalizers, namely of MWT - CMA - DFE, FMWT - CMA - DFE and DMWT - CAM - DFE, are proposed in this paper, the computational complexities of various kinds of equalizer are analyzed. Finally, in order to verify the effect of orthogonal multi-wavelet transform location on the equalizer performance, simulations are performed with underwater acoustic channel. The simulation results show that DMWT - CAM - DFE has faster the convergence speed and the ability to track the time-varying channel, compared with the CMA - DFE, MWT - CMA - DFE and FMWT -CMA-DFE. Moreover, the eye diagrams after equalization performs no phase rotation for DMWT - CAM - DFE.

\section{References}

[1] Sur Sammrendra Nath,Bera Rabindranath,Maji Bansibadan, Decision feedback equalization for MIMO systems, Advances in Intelligent Systems and Computing, 343:205-212, 2015.

[2] Iqbal Naveed, Zerguine Azzedine, Al-Dhahir Naofal. Decision Feedback Equalization using Particle Swarm Optimization. Signal Processing, 108: 1-12, 2015.

[3] Han Yingge, Guo Yecai, Li Baokun, Zhou Qiaoxi. Momentum term and orthogonal wavelet-based blind equalization algorithm. Journal of System Simulation, 20(6):1559-1562, 2008.

[4] Yang Chao, Guo Yecai. A OWT-LE+DD Algorithm Based on Orthogonal Wavelet Transform.ACTA ARMAENTARII, 31(2): 189-203, 2010.

[5] Guo Yecai, Liu Zhenxing. Blind Equalization Algorithms Based on Balance Orthogonal Multi-wavelet Transform.ACTA ARMAENTARII, 31(32): 79-284, 2010.

[6] Huang Lu Rui.Adaptive equalization algorithm based on wavelet packlet transform. Acta Electronica Sinica, 31(8): 1205-1208, 2003.

[7] Jinhun lei, Jingli Li. The research of wavelet transform blind equalization algorithm based on ant colony optimization. International Journal of Control and Automation, 7(5): 365-378, 2014. 
[8] Jalil Amir Minayi, Amindavar Hamidreza, Cances Jean-pierre.Blind equalization in wavelet domain, In Proceedings of the 2008 IEEE International Symposium on Wireless Communication Systems (ISWCS'08), 513-517, 2008.

[9] I. Daubechis, "the lectures on wavelet", Philadelphia, Society for Industrial and Applied Mathematics, 2008.

[10]Attallah S, Najin M. On the convergence enhancement of the wavelet transform based LMS, Acoustics, Speech, and Signal Processing, Detroit: IEEE, 973-976, 1995.

[11]Yang Yoon Gi, Lee Chang Su, Yang Soo Mi. Constant modulus algorithm with reduced complexity employing DFT domain fast filtering. IEICE Transactions Communications, E93-B(7): pages 1974-1979, July 2010. 